\title{
Effect of Holistic Approach Program on Mental Health among Adolescents of Barangay Dita, City of Santa Rosa, Laguna
}

\author{
Glena Fe A. Yapchulay-Alcabasa and Mary Jane Botabara-Yap \\ Adventist University of the Philippines
}

\begin{abstract}
Latest studies have shown growing concerns on the adolescents' mental health problems specifically depression, anxiety and stress. This study utilized one group pre-test post-test descriptive design that aims to determine the prevalence of mental health problems and to determine the effect of holistic approach program to adolescents at risk. Two-phase sampling technique was applied. Initial sampling was done to determine those who have mental health problems among the population. Using modified and translated DASS-21, result revealed an over-all prevalence of 89\%. Second-phase sampling included the 32 adolescents with mental health problems and underwent holistic approach intervention program. The specific prevalence showed $93.75 \%$ for depression, $84.38 \%$ for anxiety and $75 \%$ for stress. The following causes of mental health problem addressed were heavy use of social media, lack of socialization and self-esteem, peer pressure, academic difficulties, unhealthy diet, lack of sleep, low spirituality, conflict with family and friends. After the 8-sessions of the intervention program, initial over-all prevalence (100\%) decreased (56.25\%); depression, anxiety, and stress decreased as well (9.34\%, 50\% and $18.75 \%)$. Prevalence of lack of self-esteem and socialization decreased $(53.12 \%$ to $18.75 \%$ ), praying time increased to $62.50 \%$. Eating junkfoods decreased (100\% to $46.87 \%$ ) and lack of sleep decreased as well (75\% to $15.62 \%)$. Peer pressure decreased (43.75\% to $18.75 \%$ ), conflicts with family and friends decreased from $65.62 \%$ to $12.5 \%$ and $62.50 \%$ to $25 \%$, respectively. Academic difficulties were resolved (62.5\% to 6.25\%). Therefore, the holistic approach intervention program was seen to be an effective tool in addressing depression, anxiety and stress among the adolescents.
\end{abstract}

Keywords: mental health, adolescents, anxiety, depression, stress, holistic approach program 